\title{
Albuminuria in Australian Aboriginal people: prevalence and associations with components of the metabolic syndrome
}

\author{
K. G. Rowley ${ }^{1}$, D.M. Iser ${ }^{1}$, J.D. Best ${ }^{1}$, K. O'Dea ${ }^{2}$, D. Leonard ${ }^{3}$, R. McDermott ${ }^{3}$ \\ ${ }^{1}$ The University of Melbourne, Department of Medicine, St Vincent's Hospital, Fitzroy, Australia \\ ${ }^{2}$ Menzies School of Health Research, Casuarina, Australia \\ ${ }^{3}$ Queensland Health, Tropical Public Health Unit, Cairns, Australia
}

\section{Abstract}

Aims/hypothesis. To examine the prevalence and associations with the metabolic syndrome of albuminuria among Australian Aboriginal people.

Methods. Early-morning urine specimens were collected as part of community-based risk factor surveys assessing the prevalence of diabetes and cardiovascular disease in eight remote communities, with a sample size of 1,075 people. Microalbuminuria was defined as urinary albumin : creatinine ratio $3.4-33.9 \mathrm{mg} / \mathrm{mmol}$, macroalbuminuria as albumin : creatinine ratio equal to or greater than $34 \mathrm{mg} / \mathrm{mmol}$. Results. There were high prevalences of microalbuminuria (men $22.2 \%$, women $26.9 \%$ ) and of macroalbuminuria (men 10.4\%, women $13.5 \%$ ). There were highly statistically significant linear associations of microalbuminuria and macroalbuminuria with increasing number of coexisting components of the metabolic syndrome (hypertension, glucose intolerance, dyslipidaemia, insulin resistance, abdominal obesity): among people with zero, one, two and three to five of these conditions, respectively, prevalence of microalbuminuria was $16 \%, 20 \%, 36 \%$ and $32 \%$ $(p<0.001)$; prevalence of macroalbuminuria was $2 \%, 6 \%, 12 \%$ and $32 \%(p<0.001)$. There were independent associations of microalbuminuria with hypertension (odds ratio, 95\% confidence inter$\mathrm{val}=2.36,1.63-3.42)$ and diabetes $(2.10,1.28-3.45)$ : macroalbuminuria was independently associated with hypertension $(6.39,3.93-10.4)$, diabetes (3.49, 1.93-6.28) and abdominal obesity (4.56, 2.40-8.64) and had a weaker association with insulin resistance (1.99, 1.12-3.54). Dyslipidaemia and impaired glucose tolerance were neither independently associated with microalbuminuria or macroalbuminuria, nor was insulin resistance or abdominal obesity independently associated with microalbuminuria.

Conclusion/interpretation. There was a strong clustering of albuminuria with components of the metabolic syndrome. Diabetes, hypertension and abdominal obesity are major contributors to high rates of albuminuria among Australian Aboriginal people. [Diabetologia (2000) 43: 1397-1403]

Keywords Microalbuminuria, Australian Aboriginal people, epidemiology, metabolic syndrome, insulin resistance.
High prevalences of albuminuria and renal disease have been reported in several Australian Aboriginal

Received: 14 June 2000 and in revised form: 20 July 2000

Corresponding author: K. G. Rowley, The University of Melbourne, Department of Medicine, St Vincent's Hospital, Fitzroy VIC 3065, Australia

Abbreviations: ACR, Albumin to creatinine ratio; HDL, high density lipoprotein; HOMA, homeostasis model assessment; OR, odds ratio; PAR \%, population attributable risk per cent; UTI, urinary tract infection. populations $[1,2]$. The incidence of end-stage renal disease in Northern Territory Aboriginal people was 925 per million in 1996 [3] and in a cohort of 374 central Australian Aboriginal people with diabetes followed from 1984 to 1991, renal disease was the most common cause of death [4]. Cardiovascular diseases remain the leading cause of death among Aboriginal and Torres Strait Islander people [5] and albuminuria is probably a risk factor for cardiovascular disease in this population as it is in others [6-8]. 
Among Aboriginal people there is a high prevalence of cardiovascular risk factors commonly associated with the metabolic syndrome [9]. Diabetes and hypertension strongly predict the presence of albuminuria in several population studies [10-14]. Some studies have indicated that abdominal obesity, insulin resistance and dyslipidaemia are also independently associated with albuminuria [2, 11, 14].

As part of diabetes and cardiovascular disease risk-factor surveys conducted in remote communities in northern and central Australia between 1995 and 1997, we examined urinary albumin excretion in community-based groups of over 1000 adult Aboriginal people. We report the prevalence of microalbuminuria and macroalbuminuria and have sought to determine the associations of albumin excretion with a range of conditions that constitute the metabolic syndrome, including insulin resistance and abdominal obesity.

\section{Subjects and methods}

The protocols described were approved by Deakin University Ethics Committee (the institution responsible at the time of the surveys), the Alice Springs Institutional Ethics Committee, the Ethics Committee of the Peninsula and Torres Strait Regional Health Authority and Cairns Base Hospital Institutional Ethics Committee.

Subject selection. Between 1995 and 1997 eight remote communities were screened for diabetes, albuminuria and other cardiovascular risk factors. These community-based surveys were carried out in three central Australian communities, one in the Kimberley region of Western Australia and four in Cape York, Queensland. All community members aged 15 and older were invited to participate. The response rates were $70 \%$ for central Australian Aboriginal people, $75 \%$ for Kimberley Aboriginal people and approximately $40 \%$ for Cape York Aboriginal people. The surveys in the Cape York area included a number of Torres Strait Islander people and these were excluded from this study. The survey sample was representative of the Australian indigenous population [15] for age and sex distribution. Further details of screening procedures and survey sample characteristics have been reported previously $[16,17]$. Urine samples, complete anthropometric data (body weight and height, waist and hip circumferences), blood pressure measurements and an oral glucose tolerance test or a previously confirmed diagnosis of diabetes were available for $92 \%$ of this survey sample and these subjects are included in the study. Pregnant women $(n=10)$ were excluded.

Laboratory and anthropometric techniques. Because 24-h urine collections were not possible for cultural and logistical reasons, the albumin to creatinine ratio (ACR) in early-morning urine samples with which it correlates well was used as a surrogate marker of albumin excretion rate [18]. Samples were kept cool before being frozen at $-20^{\circ} \mathrm{C}$ or colder and were assayed within 2 months (usually less than 1 month) of collection. The stability of albumin during freeze-thawing has been questioned but the differences reported after freeze-thawing are generally small and occur in a non-differential manner [19]. Although field conditions require freezing of samples, this process did not greatly influence our conclusions. Urinary albumin concentration was measured using immunonephelometry [Kallestadt QM300 or Beckman 360 Array nephelometers; interassay coefficient of variance (CV) 3-5\%]. Urinary creatinine concentration was measured using an alkaline picrate method (Olympus AU800 autoanalyser; interassay CV 2\%). Microalbuminuria was defined as ACR in the range of 3.4 to less than $34 \mathrm{mg} / \mathrm{mmol}$ and macroalbuminuria was defined as an ACR of $34 \mathrm{mg} / \mathrm{mmol}$ or more, recommended by the United States National Kidney Foundation [20] and also appropriate for a population with a linear body build and a relatively low lean mass for a given BMI [21-24]. For samples collected in central Australia, suspected urinary tract infection (UTI) was diagnosed by urinalysis using reagent strips (N-Multistix SG, Bayer Diagnostics, Mulgrave, VIC, Australia) and these samples were sent for further microbiological examination to confirm the diagnosis. The presence of haematuria was also investigated using these reagent strips.

Plasma glucose, triglycerides and high density lipoprotein (HDL) cholesterol (after precipitation of other lipoproteins with $15 \%$ polyethylene glycol MW 6000) were measured using standard enzymatic techniques (Boehringer-Mannheim reagents; interassay $\mathrm{CV}<7 \%$ ). Insulin was measured by radioimmunoassay using a specific antibody (Linco Research, St. Charles Mo., USA; interassay CV 9-13\%). Insulin resistance was estimated from fasting glucose and insulin concentrations using homeostasis model assessment (HOMA) [25]. Blood pressure was measured with a Dinamap automated blood pressure monitor (Critikon Inc., Tampa Fla., USA). Body weight was measured to $0.1 \mathrm{~kg}$ and height, waist, and hip circumferences to $0.1 \mathrm{~cm}$. For categorising conditions that constitute the metabolic syndrome, dyslipidaemia was defined as the combination of increased fasting triglycerides $(\geq 2.0 \mathrm{mmol} / \mathrm{l})$ and low HDL cholesterol $(<0.91 \mathrm{mmol} / \mathrm{l})$, hypertension was defined as systolic blood pressure $\geq 140 \mathrm{mmHg}$ and/or diastolic blood pressure $\geq 90 \mathrm{mmHg}$ and/or current antihypertensive medication [26]. Subjects were categorised as "insulin resistant" if in the highest quartile of HOMA insulin resistance and cut-offs for high waist-to-hip ratio were 1.0 for men and 0.85 for women [27]. Diabetes and impaired glucose tolerance (IGT) were defined using World Health Organization (WHO) criteria based on a 75 -g oral glucose tolerance test.

Statistical analyses. Differences in prevalence of microalbuminuria and macroalbuminuria across strata were tested using the chi-squared statistic for independence $\left(\chi_{\text {indep }}^{2}\right)$ or the Mantel-Haenzel chi-squared statistic for linear trend $\left(\chi_{\text {trend }}^{2}\right)$ as appropriate. Differences in mean levels of continuous variables across categories of ACR were tested by ANOVA using the General Linear Modelling function on SPSS 9.0 (SPSS Inc., Chicago Il., USA). Values for triglycerides and insulin resistance were log-transformed for these analyses and data are presented as geometric mean. Multinomial logistic regression analysis was used to determine odds ratios for categorical predictors of microalbuminuria and macroalbuminuria: blood pressure (hypertensive vs normotensive), glucose tolerance (non-diabetic versus diabetic), dyslipidaemia, BMI $(<25,25-$ $70,>30 \mathrm{~kg} / \mathrm{m}^{2}$ ), waist-to-hip ratio (normal vs high), insulin resistance (quartiles of HOMA insulin resistance), age (15-24 years, 25-34 years, 35-44 years and 45 years or more), sex and geographic location (central Australia, Kimberley, Cape York). Population attributable risk per cent was calculated using the formula: $100 \times \mathrm{P}_{\mathrm{e}}(\mathrm{OR}-1) /\left(\mathrm{P}_{\mathrm{e}}(\mathrm{OR}-1)+1\right)$, where $\mathrm{P}_{\mathrm{e}}$ is the proportion of the population exposed to the predictor variable of interest [28]. differences were considered statistically significant at $p<0.05$. 
Table 1. Prevalence of elevated urinary ACR stratified by age, sex and geographic location $(n=1075)$

\begin{tabular}{lccc}
\hline & $n$ & $\begin{array}{c}\text { Prevalence (\%) } \\
\text { Microalbuminuria }\end{array}$ & $\begin{array}{l}\text { Macro- } \\
\text { albuminuria }\end{array}$ \\
\hline Age group & & & \\
15-24 years & 302 & 16.6 & 5.0 \\
25-34 years & 290 & 22.4 & 9.0 \\
35-44 years & 181 & 24.9 & 14.4 \\
45 years + & 302 & 35.4 & 20.9 \\
$p$-value & & $<0.001$ & $<0.001$ \\
Sex & & & \\
Male & 473 & 22.2 & 10.4 \\
Female & 602 & 26.9 & 13.5 \\
$p$-value & & 0.076 & 0.122 \\
Location & & & \\
Central Australia & 794 & 25.1 & 12.5 \\
Kimberley & 174 & 21.3 & 14.4 \\
Cape York & 107 & 29.0 & 5.6 \\
$p$-value & & 0.334 & 0.075 \\
\hline
\end{tabular}

\section{Results}

There was a strong linear trend towards increasing prevalence with increasing age for both microalbuminuria $\left(\chi_{\text {trend }}^{2}=48.0, p<0.001\right)$ and macroalbuminuria $\left(\chi_{\text {trend }}^{2}=39.4, p<0.001\right.$; Table 1$)$. Men and women had similar prevalences of microalbuminuria $\left(\chi_{\text {in- }}^{2}\right.$ dep $^{2}=3.2, p$ not significant) and macroalbuminuria $\left(\chi_{\text {indep }}^{2}=2.4, p\right.$ not significant; Table 1$)$. There were no significant differences in the crude prevalence of microalbuminuria $\left(\chi_{\text {indep }}^{2}=2.2, p\right.$ not significant $)$ or macroalbuminuria ( $\chi^{2}$ indep $=5.2, p$ not significant $)$ according to geographic location (Table 1 ).

When continuous variables were stratified by categories of ACR, there were strong associations of systolic and diastolic blood pressure with increasing ACR (Table 2). The exclusion of patients currently prescribed antihypertensive medication $(n=70)$ did not substantially alter these associations (data not shown). Body mass index varied significantly across ACR categories: mean (95\% CI) BMI was 25.4 (24.9 - 26.0), $27.7(26.9-28.5)$ and $28.5(27.4-29.6) \mathrm{kg} / \mathrm{m}^{2}$ for normal, microalbuminuric and macroalbuminuric people respectively $(p<0.001)$. Waist-to-hip ratio varied significantly across ACR categories (Table 2 ), with successive increases in mean value with greater degree of albuminuria. Fasting plasma triglycerides varied significantly across categories of ACR, with significantly elevated concentration in macroalbuminuric people compared to normal and microalbuminuric people (Table 2) but HDL cholesterol was only weakly associated with albuminuria. Insulin resistance estimated from HOMA was significantly greater with increasing ACR category (Table 2).

When categorical variables were stratified by categories of ACR, there was a statistically significant increase in the age-adjusted prevalence (using the overall survey sample as the reference population) of diabetes with worsening degrees of albuminuria $\left(\chi_{\text {trend }}^{2}=74.2, p<0.001\right.$; Table 2$)$. The prevalence of IGT did not vary according to ACR category $\left(\chi_{\text {trend }}^{2}=0.4, p\right.$ not significant; Table 2$)$. When glucose tolerance was defined according to fasting plasma glucose concentration using American Diabetes Association (ADA) criteria [29], the age-adjusted prevalence of impaired fasting glucose was $4.3 \%, 5.1 \%$ and $4.5 \%$ in normal, microalbuminuric and macroalbuminuric people respectively $\left(\chi_{\text {trend }}^{2}=0.03\right.$, $p=0.875)$ and the prevalence of diabetes was $6.7 \%$, $17.9 \%$ and $34.1 \%$ in normal, microalbuminuric and macroalbuminuric people respectively $\left(\chi_{\text {trend }}^{2}=76.1\right.$, $p<0.001)$. Among central Australian Aboriginal people, the presence of 'moderate' or greater degrees of haematuria ( $>80$ cells/ $\mu$ l) upon dipstick analysis was considered to indicate glomerulonephritis. The prevalence of haematuria was $8.5 \%, 17.6 \%$ and $17.2 \%$ in normal, microalbuminuric and macroalbuminuric people respectively $\left(\chi_{\text {trend }}^{2}=13.3, p<0.001\right)$. Stratification of the survey sample by the presence or absence of haematuria did not greatly alter the associations with the variables shown in Table 2 (data not shown). We identified 49 confirmed cases of UTI ( 2 men, 47 women) among central Australian Aboriginal people. The prevalence of microalbuminuria and macroalbuminuria was similar in those women with and without UTI (data not shown).

Table 2. Blood pressure, waist-to-hip ratio, plasma lipids, insulin resistance and glucose tolerance stratified by ACR category

\begin{tabular}{|c|c|c|c|c|}
\hline & \multicolumn{3}{|l|}{ ACR category } & \multirow[t]{2}{*}{$p$-value } \\
\hline & Normal & Microalbuminuria & Macroalbuminuria & \\
\hline$n$ & 678 & 267 & 130 & \\
\hline Diastolic BP, mmHg & $69(68-69)$ & $71(70-73)$ & $80(78-82)$ & $<0.001$ \\
\hline Waist-to-hip ratio & $0.90(0.89-0.90)$ & $0.92(0.91-0.93)$ & $0.96(0.94-0.97)$ & $<0.001$ \\
\hline Triglycerides, mmol/1 & $1.7(1.6-1.8)$ & $2.0(1.8-2.1)$ & $2.5(2.2-2.7)$ & $<0.001$ \\
\hline HDL cholesterol, mmol/l & $0.91(0.89-0.93)$ & $0.87(0.84-0.90)$ & $0.87(0.83-0.91)$ & 0.023 \\
\hline Diabetes \% & 9.6 & 21.5 & 39.0 & $<0.001$ \\
\hline
\end{tabular}

Data are estimated marginal mean (95\% confidence interval) adjusted for age, sex and geographic location except IGT and diabetes, which are age-adjusted prevalence 
A

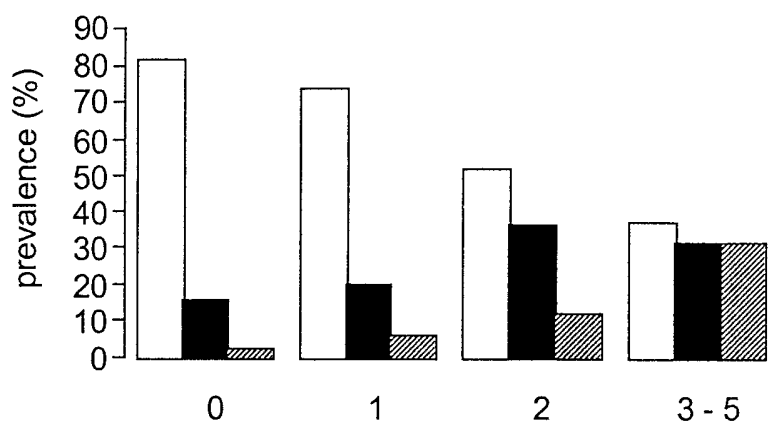

B

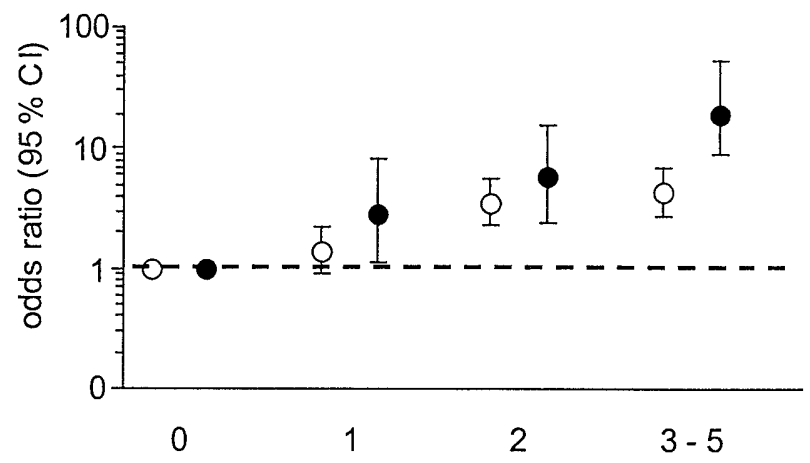

coexisting components of the metabolic syndrome

Fig.1. A Prevalence of normal ACR $(\square)$, microalbuminuria (ם) and macroalbuminuria (․․․ stratified by the number of coexisting components of the metabolic syndrome (hypertension, abnormal glucose tolerance, dyslipidaemia, insulin resistance and abdominal obesity). For people with zero, one, two and three or more components respectively, $n=312,292,209$ and 244; $\mathbf{B}$ odds ratios for microalbuminuria $(O)$ and macroalbuminuria (O) by number of coexisting components of the metabolic syndrome

The prevalence of albuminuria was examined according to the number of coexisting components of the metabolic syndrome as defined above (Fig.1). The proportion of the sample with zero, one, two and more than two of these conditions was $29.5 \%$, $27.6 \%, 19.8 \%$ and $23.0 \%$ respectively. Among people with only one of these components, $18.8 \%$ were hypertensive, $7.2 \%$ were glucose intolerant (diabetes or IGT), $32.2 \%$ were dyslipidaemic, $6.5 \%$ were insulin resistant and $35.3 \%$ had abdominal obesity. There was a progressive decrease in the proportion of people with normal ACR with increasing number of coexisting components (Fig.1) and a corresponding increase in the prevalence of microalbuminuria $\left(\chi_{\text {trend }}^{2}=64.1, \quad p<0.001\right)$ and macroalbuminuria $\left(\chi_{\text {trend }}^{2}=110, p<0.001\right)$. The risk of macroalbuminuria was significantly increased in the presence of one or more components of the metabolic syndrome, as indicated by odds ratios significantly greater than one (Fig. 1B). The risk of microalbuminuria was significantly increased in the presence of two or more components of the metabolic syndrome. Even when no components of the metabolic syndrome were present, there was however, a substantial prevalence of microalbuminuria.

Independent odds ratio (OR; derived from multinomial logistic regression as described above) and population attributable risk per cent (PAR \%; see above) for components of the metabolic syndrome were calculated (Table 3). Hypertension was associated with a statistically significant increased risk of albuminuria $(p<0.001)$, with significantly increased OR for microalbuminuria and macroalbuminuria. Diabetes was associated with increased risk of albuminuria independently of other factors $(p<0.001)$, with greater risk of microalbuminuria and macroalbuminuria in diabetic people. Impaired glucose tolerance was not a statistically significant predictor of microalbuminuria or macroalbuminuria and was excluded from the final model. Dyslipidaemia was not independently associated with albuminuria $(p=0.181)$. Obesity defined by BMI criteria was not a significant risk factor and was omitted from the final model. High waist-to-hip ratio was independently associated with albuminuria $(p<0.001)$, with a significantly higher risk of macroalbuminuria in abdominally obese people $(p<0.001)$. The risk of microalbuminuria associated with abdominal obesity was not statistically significant ( $p=0.126)$. Overall, insulin resistance did not reach statistical significance as an independent risk factor for albuminuria $(p=0.073)$. Results of multinomial logistic regression analyses remained substantially unchanged after stratification of the survey sample by diabetes or hypertension status. In terms of PAR \%, hypertension, diabetes and ab-

Table 3. Independent odds ratio (OR; adjusted for all other factors) and population attributable risk per cent (PAR \% ) for components of the metabolic syndrome in relation to risk of microalbuminuria and macroalbuminuria

\begin{tabular}{|c|c|c|c|c|c|}
\hline \multirow[t]{2}{*}{ Condition } & \multicolumn{3}{|c|}{ Microalbuminuria } & \multicolumn{2}{|c|}{ Macroalbuminuria } \\
\hline & Prevalence & OR $(95 \% \mathrm{CI})$ & PAR \% & OR $(95 \% \mathrm{CI})$ & PAR \% \\
\hline Hypertension & $25.6 \%$ & $2.4(1.6-3.4)$ & $26(14-38)$ & $6.3(3.9-10.2)$ & $58(42-70)$ \\
\hline Dyslipidaemia & $35.6 \%$ & $1.1(0.8-1.6)$ & $4(<0-17)$ & $1.6(1.0-2.6)$ & $17(<0-36)$ \\
\hline Abdominal obesity & $37.4 \%$ & $1.3(0.9-1.9)$ & $11(<0-25)$ & $4.7(2.5-8.8)$ & $58(35-75)$ \\
\hline Insulin resistance ${ }^{\mathrm{a}}$ & & $1.8(1.1-3.0)$ & & $1.2(0.6-2.6)$ & \\
\hline
\end{tabular}

OR derived from multinomial logistic regression; PAR \% calculated according to reference [28]

${ }^{a}$ Highest quartile of HOMA insulin resistance compared to lowest quartile 
dominal obesity were major independent contributors to the prevalence of macroalbuminuria in this population. Hypertension accounted for about one quarter of the subjects with microalbuminuria, diabetes for less than $20 \%$ and other conditions of the metabolic syndrome did not contribute significantly.

Among the central Australian group of people for whom the presence of UTI was determined (prevalence of UTI $=7.1 \%$ ), the presence of UTI was not statistically significantly associated with albuminuria and was omitted from the final model. Haematuria at levels indicative of glomerulonephritis (see above) was strongly and independently associated with both microalbuminuria $(\mathrm{OR}, 95 \% \mathrm{CI}=3.3,1.9$ $-5.6)$ and macroalbuminuria $(5.0,2.4-10.6)$. The inclusion or exclusion of haematuric people from the analysis, however, did not substantially alter the risks associated with other variables (data not shown).

\section{Discussion}

Our results indicate that albuminuria among Aboriginal people in Central Australia, the Kimberley and Cape York occurs with a very high prevalence in non-diabetic and diabetic people. Albuminuria clustered strongly with characteristics of the metabolic syndrome: hypertension, glucose intolerance, dyslipidaemia, abdominal obesity (all of which were highly prevalent in this population) and insulin resistance. Moreover, microalbuminuria was highly prevalent even in the absence of any of these conditions. In multivariate analyses, microalbuminuria was independently associated only with hypertension and diabetes: macroalbuminuria showed highly statistically significant, independent associations with hypertension, diabetes and abdominal obesity but no statistically significant association with insulin resistance. Dyslipidaemia and IGT were not independently associated with albuminuria. The data are consistent with the high rates of albuminuria and renal disease in other Aboriginal populations in Australia [1, 2]. Our results are also quantitatively and qualitatively consistent with other population-based studies in the role of blood pressure, diabetes, IGT and glomerulonephritis as independent risk factors for albuminuria $[2,11,14,30]$.

A role for total and abdominal adiposity as a risk factor for albuminuria has been reported inconsistently among European, American and Australian populations $[2,8,11,14,31-34]$. In our survey sample, ACR was clearly associated with waist-to-hip ratio but only weakly or not at all with BMI in itself. Prevention of obesity would, however, clearly reduce the risk of albuminuria through concomitant prevention of hypertension, diabetes and other aspects of the metabolic syndrome.
Insulin resistance and hyperinsulinaemia have been linked previously with albuminuria. Fasting hyperinsulinaemia was independently associated with increased ACR in another Aboriginal population [2] and insulin resistance was statistically significantly associated with ACR in the non-diabetic population of the Insulin Resistance Atherosclerosis Study [11]. In other studies, albuminuria in patients with Type II (non-insulin-dependent) diabetes mellitus was associated with greater insulin resistance than in normalbuminuric diabetic patients [12], whereas in hypertensive patients, a positive association between insulin response to oral glucose and albumin excretion rate was observed [35]. In contrast to these studies, no independent association of fasting hyperinsulinaemia with albuminuria was found in a populationbased study of European people [14] and the authors concluded that microalbuminuria was "a complication of hypertension and diabetes but not an integral part of the insulin resistance syndrome". In our study, insulin resistance was clearly higher with worsening degree of albuminuria. This trend was, however, largely explained by higher prevalences of diabetes among people with a greater ACR and multivariate analysis showed an independent relation between insulin resistance and albuminuria that was at best weak. Similar negative conclusions were reached in a study of several other indigenous populations [36].

Primary causes of renal disease leading to transplantation or dialysis among Aboriginal patients in the Northern Territory, Western Australia and Queensland from 1993 to 1997 (the period during which the present cross-sectional surveys were carried out) included diabetes (46\%), hypertension $(5 \%)$ and glomerulonephritis (29\%) [37]. Apart from the expense, treatment of end-stage renal disease with dialysis or transplantation has severe detrimental social effects on Aboriginal people [38, 39] and prevention of the initiation and progression of albuminuria is clearly a better method of dealing with this issue. Regardless of the exact causal associations between constituent components of the metabolic syndrome and albumin excretion, interventions to prevent obesity, diabetes and hypertension based on improved social conditions, diet and exercise are likely to contribute to the prevention of this major cause of premature morbidity and mortality among Aboriginal people.

Acknowledgements. This study was funded by a grant from the National Health and Medical Research Council of Australia (NHMRC No 954605). Some of this data has been presented and published in abstract form at the Annual Scientific Meeting of the Australian Diabetes Society, Melbourne, September 1995. K. G. Rowley is an NHMRC Public Health Research and Development Committee Postdoctoral Fellow. The authors particularly thank S. Knight and the Councils, members and Health Service staff of the communities involved in this study, G. Dragicevic for expert laboratory assistance and data colla- 
tion, C. Karschimkus and O. Strommer for technical assistance, P. Burgess and M. Cincotta for assistance with sample processing in the field, M. Daniel and E. Villanueva for statistical advice and P. Lee for helpful discussions on the interpretation of data. The dialysis data cited were supplied on request by L. Excel of the Australia and New Zealand Dialysis and Transplant Registry.

\section{References}

1. Guest CS, Ratnaike SR, Larkins RG (1993) Albuminuria in Aborigines and Europids of south-eastern Australia. Med J Aust 159: 335-338

2. Hoy WE, Mathews JD, McCredie DA et al. (1998) The multidimensional nature of renal disease: Rates and associations of albuminuria in an Australian Aboriginal community. Kidney Int 54: 1296-1304

3. Spencer JL, Silva DT, Snelling P, Hoy WE (1998) An epidemic of renal failure among Australian Aboriginals. Med J Aust 168: 537-541

4. Phillips CB, Patel MS, Weeramanthri TS (1995) High mortality from renal disease and infection in Aboriginal central Australians with diabetes. Aust J Public Health 19: 482-486

5. Thomson NJ (1991) Recent trends in Aboriginal mortality. Med J Aust 154: 235-239

6. Neil A, Hawkins M, Potok M, Thorogood M, Cohen D, Mann J (1993) A prospective population-based study of microalbuminuria as a predictor of mortality in NIDDM. Diabetes Care 16: 996-1003

7. Yudkin J, Forrest RD, Jackson CA (1988) Microalbuminuria as a predictor of vascular disease in non-diabetic subjects - Islington Diabetes Survey. Lancet ii: 530-532

8. Haffner SM, Stern MP, Gruber KK, Hazuda HP, Mitchell BD, Patterson JK (1990) Microalbuminuria. Potential marker for increased cardiovascular risk factors in nondiabetic subjects? Arteriosclerosis 10: 727-731

9. Rowley KG, Best JD, McDermott R, Green EA, Piers LS, O'Dea K (1997) Insulin resistance syndrome in Australian Aboriginal people. Clin Exp Pharmacol Physiol 24: 776-781

10. Mogensen CE (1999) Microalbuminuria, blood pressure and diabetic renal disease: origin and development of ideas. Diabetologia 42: 263-285

11. Mykkänen L, Zaccaro DJ, Wagenknecht LE, Robbins DC, Gabriel M, Haffner SM (1998) Microalbuminuria is associated with insulin resistance in nondiabetic subjects. The Insulin Resistance Atherosclerosis Study. Diabetes 47: 793-800

12. Niskanen L, Laakso M (1993) Insulin resistance is related to albuminuria in patients with Type II (non-insulin-dependent) diabetes mellitus. Metabolism 42: 1541-1545

13. Nosadini R, Cipollina MR, Solini A et al. (1992) Close relationship between microalbuminuria and insulin resistance in essential hypertension and non-insulin dependent diabetes mellitus. J Am Soc Nephrol 3: S56-S63

14. Jager A, Kostense PJ, Nijpels G, Heine RJ, Bouter LM, Stehouwer CDA (1998) Microalbuminuria is strongly associated with NIDDM and hypertension, but not with the insulin resistance syndrome: the Hoorn study. Diabetologia 41: 694-700

15. McLennan W, Madden R (1997) The Health and Welfare of Australia's Aboriginal and Torres Strait Islander Peoples. Australian Bureau of Statistics no. Australian Government Publishing Service, Canberra
16. Rowley KG, Gault A, McDermott RG, Knight S, McLeay T, O'Dea K (2000) Reduced prevalence of impaired glucose tolerance and no change in prevalence of diabetes despite increasing BMI among Aboriginal people from a group of remote homeland communities. Diabetes Care 23: 898-904

17. McDermott R, Rowley KG, Lee AJ, Knight S, O'Dea K (2000) Increase in the prevalence of obesity and diabetes and decrease in plasma cholesterol in a central Australian Aboriginal community. Med J Aust 172: 480-484

18. Chaiken RL, Khawaja R, Bard M, Eckert-Norton M, Banerji MA, Lebovitz HE (1997) Utility of untimed urinary albumin measurements in assessing albuminuria in black NIDDM subjects. Diabetes Care 20: 709-713

19. Shield JPH, Hunt LP, Morgan JE, Pennock CA (1995) Are frozen urine samples acceptable for estimating albumin excretion in research? Diabet Med 12: 713-716

20. Bennett PH, Haffner S, Kasiske BL et al. (1995) Screening and management of microalbuminuria in patients with diabetes mellitus: recommendations to the scientific advisory board of the National Kidney Foundation from an ad hoc committee of the council on diabetes mellitus of the National Kidney Foundation. Am J Kidney Dis 25: 107-112

21. Mogensen CE, Vestbo E, Poulsen PL et al. (1995) Microalbuminuria and potential confounders. Diabetes Care 18: 572-581

22. Gupta RC, Gupta U (1986) Urinary protein/creatinine index. Clin Chem 32: 914

23. American Diabetes Association (1994) Consensus development conference on the diagnosis and management of nephropathy in patients with diabetes mellitus. Diabetes Care 17: 1357-1361

24. Viberti GC, Mogensen CE, Passa P, Bilous R, Mangili R (1994) St. Vincent declaration, 1994: guidelines for the prevention of diabetic renal failure. In: Mogensen CE (ed) The kidney and hypertension in diabetes mellitus. Kluwer, Boston, pp 515-527

25. Matthews DR, Hosker JP, Rudenski AS, Naylor BA, Treacher DF, Turner RC (1985) Homeostasis model assessment: insulin resistance and b-cell function from fasting plasma glucose and insulin concentrations in man. Diabetologia 28: 412-419

26. Joint National Committee on Detection, Evaluation and Treatment of High Blood Pressure (1993) The fifth report of the Joint National Committee on Detection, Evaluation and Treatment of High Blood Pressure. Arch Intern Med 153: $154-183$

27. James WPT (1996) The epidemiology of obesity. In: Chadwick D, Cardew G (eds) The origins and consequences of obesity. Wiley, Chichester, pp 1-16

28. Basu S, Landis JR (1995) Model-based estimation of population attributable risk under cross-sectional sampling. Am J Epidemiol 142: 1338-1343

29. The Expert Committee on the Diagnosis and Classification of Diabetes Mellitus (1997) Report of the Expert Committee on the Diagnosis and Classification of Diabetes Mellitus. Diabetes Care 20: 1183-1197

30. Hoy W, Jim S, Warrington W, Light A, Megill D (1996) Urinary findings and renal function in adult Navajo Indians and associations with type 2 diabetes. Am J Kidney Dis 28: 339-349

31. Goetz FC, Jacobs DR, Chavers B, Roel J, Yelle M, Sprafka JM (1997) Risk factors for kidney damage in the adult population of Wadena, Minnesota. Am J Epidemiol 145: 91-102

32. Bruno G, Cavallo-Perin P, Bargero G et al (1996) Prevalence and risk factors for micro- and macroalbuminuria in 
an Italian population-based cohort of NIDDM subjects. Diabetes Care 19: 43-47

33. Klein R, Klein BEK, Moss SE (1997) Is obesity related to microvascular and macrovascular complications in diabetes? Arch Int Med 157: 650-656

34. Smulders YM, Rakic M, Stehouwer CDA, Weijers RNM, Slaats EH, Silberbusch J (1997) Determinants of progression of microalbuminuria in patients with NIDDM. Diabetes Care 20: 999-1005

35. Bianchi S, Bigazzi R, Valtriani C et al (1994) Elevated serum insulin levels in patients with essential hypertension and microalbuminuria. Hypertension 23: 681-687

36. Hodge AM, Dowse GK, Zimmet PZ (1996) Microalbuminuria, cardiovascular risk factors, and insulin resistance in two populations with a high risk of Type 2 diabetes mellitus. Diabet Med 13: 441-449

37. Data supplied on request by the ANZDATA Registry. From: Disney APS, Russ GR, Walker R et al (eds) Australia and New Zealand Dialysis and Transplant Registry, Adelaide, South Australia.

38. Bennett E, Manderson L, Kelly B, Hardie I (1995) Cultural factors in dialysis and renal transplantation among Aborigines and Torres Strait Islanders in North Queensland. Aust J Public Health 19: 610-615

39. Willis J (1995) Fatal attraction: do high-technology treatments for end stage renal disease benefit Aboriginal patients in central Australia? Aust J Public Health 19: 603-609 